la revue La revue pour l'histoire du CNRS

POUR LHISTORE DU CNRS $\quad 24 \mid 2009$

Soixante-dixième anniversaire du CNRS

\title{
Le jeu, la règle et le compas
}

\section{François Sauvageot}

\section{(2) OpenEdition}

Journals

Édition électronique

URL : https://journals.openedition.org/histoire-cnrs/9128

DOI : 10.4000/histoire-cnrs.9128

ISSN : 1955-2408

Éditeur

CNRS Éditions

Édition imprimée

Date de publication : 5 octobre 2009

ISSN : 1298-9800

Référence électronique

François Sauvageot, "Le jeu, la règle et le compas », La revue pour l'histoire du CNRS [En ligne], 24 I 2009, mis en ligne le 05 octobre 2009, consulté le 20 mai 2021. URL : http://journals.openedition.org/ histoire-cnrs/9128; DOI : https://doi.org/10.4000/histoire-cnrs.9128

Ce document a été généré automatiquement le 20 mai 2021.

Comité pour l'histoire du CNRS 


\title{
Le jeu, la règle et le compas
}

\author{
François Sauvageot
}

1 Avant de parler de jeux, il faut parler des règles! Celles du jeu, bien sûr. L'épistémologue et psychologue Jean Piaget (1896-1980) rapporte, lors d'une entrevue avec des membres de l'association française des chercheurs en didactiques, l'expérience suivante: " [On demandait] combien on peut mettre de points entre deux points limites. C'était très drôle comme progression avec l'âge... les petits vous disaient qu'on peut en mettre une dizaine, pas plus; ensuite c'est passé à 30, ensuite à 100. Mais ce n'est qu'à partir de 11- 12 ans qu'ils commençaient à dire: on peut en mettre tant qu'on veut.» On trouve des choses amusantes au point de vue de l'infini. Voilà un exemple : l'expérience consiste à tracer une ligne (sur un objet rectangulaire par exemple) à partir de laquelle l'objet va chuter, la ligne d'équilibre. Problème : avec un rectangle, combien peut-on trouver de ces lignes? Réponse: 4 . En revanche pour un cercle, c'était une infinité. Alors on reprenait le rectangle en disant: «Place-le comme tu veux.» Et au moment où il se mettait à le tourner : "Ah, je peux le tourner? Alors c'est la même chose, il y en a un nombre infini. Mais ça, c'est à 11-12 ans." François Sauvageot voit là l'essence même des mathématiques : remettre en question les règles, les changer, en accepter de nouvelles, les tester, les pousser dans leurs derniers retranchements. Et au fond, quelle différence y a-t-il avec le jeu?

Des modèles pour agir et comprendre

2 À l'école, les énoncés qui s'ancrent dans le monde des adultes tout en le simplifiant courent le risque d'être rejetés par les enfants parce qu'ils sont en contradiction avec leur propre vécu. Il faut pouvoir sortir du monde de l'enfant, tout en lui restant accessible. Et le jeu le permet mais pas de n'importe quelle façon! Il ne faut pas gommer toute l'étape qui consiste à montrer qu'il vient du réel, qu'il en est un modèle et qu'il est obtenu par simplification. Les mathématiques et le raisonnement hypothético- déductif ne livrent des clefs des problèmes de la réalité que si ce lien est fait. Lors de séances d'animations, on peut montrer comment un jeu est relié à des problèmes concrets. C'est le cas du jeu du baguenodier ${ }^{1}$ qui amène à comprendre la façon dont sont numérotés les disques durs. Pourtant, même dans des cas élémentaires, l'action est le plus souvent en avance sur la conceptualisation. Autrement dit, on peut arriver à faire des choses avant de pouvoir dire ce qu'on fait et comment. C'est vrai en 
mathématiques, par exemple en numération: on sait ce qu'est un nombre avant de savoir dénombrer, classer, ordonner, compter, avant que la notion même de nombre ne soit acquise.

Mathématiques, hasard et justice

Les notions de partage équitable (juste) qui apparaissent très tôt à l'école et ailleurs, confrontent les individus à la maittrise du hasard, ou tout au moins à sa compréhension. Il faut accepter le hasard, s'accommoder de sa présence et ne pas chercher systématiquement les recettes qui le réduiraient à des causes purement déterministes. L'enjeu est de taille car les probabilités et leur langage ne sont pas perçus simplement. La plupart de nos concitoyen-ne-s en ont des idées fausses, qu'ils soient des personnalités politiques, qu'ils travaillent dans la banque ou l'assurance, ou même qu'ils évoluent dans un univers scientifique (et parfois même mathématique). Intéressons-nous aux enfants qui jouent aux billes dans la cour. Supposons qu'à chaque fois, chacun d'eux peut gagner la bille de son adversaire ou perdre la sienne, et ce une fois sur deux. Un enfant n'ayant presque plus de billes ira en voir un autre et lui proposera de s'associer à lui en lui donnant ses billes. Au bout d'un certain temps, ils stoppent leur association pour partir en vacances. Question : comment se répartissentils les billes? Plusieurs réponses sont possibles: partager les gains ou les pertes au prorata de ce que chacun a amené, ou à parts égales. "Une classe d'enfants handicapés à qui je posais la question m'a instantanément répondu que le partage se faisait à parts égales mais sur l'ensemble des billes, pas sur les gains ", explique François Sauvageot. Avant de préciser le regard submergé par l'émotion : «La justification était sans appel : c'est ça une association!» En fait, cette situation fait réfléchir à la notion de partage, mais aussi de mariage. C'est pourquoi aucune réponse n'est meilleure qu'une autre. Mieux, le point de vue de chaque individu évolue au cours de sa vie. On touche ici à la polysémie des mathématiques. Tous les calculs faits dans une situation donnée sont exacts et fournissent une unique réponse, mais leur sens est variable. Le chemin emprunté est bien balisé, mais l'endroit où il conduit reste aléatoire, sans pour autant être arbitraire. Le mathématicien et physicien John von Neumann (1903-1957) a dit un jour : «If people do not believe that mathematics is simple, it is only because they do not realize how complicated life is. $»^{2}$ Ce que François Sauvageot se plait à reprendre et à réinterpréter par: "Les mathématicien-ne-s ne viennent pas pour rendre simple ce qui est complexe, ni pour vendre des recettes, mais pour que chacun puisse regarder la vie au fond des yeux!»

Texto

Pour se convaincre du rôle social des mathématicien-ne-s en science populaire et dans l'enseignement primaire ou secondaire, on peut lire :

- attirer les élèves vers les mathématiques, le rôle des mathématiciens

http://www.math.sciences.univnantes.fr/ sauvageo/Recherche/

Actes/

08Montreal.pdf

- les yeux d'Uranie ou le regard et les mots des mathématiciens

http://www.math.sciences.univnantes.fr/ sauvageo/Recherche/

Actes/

08JulesVerne.pdf

Sur une autre expérience de Jean Piaget à propos de l'action précédant la conceptualisation, on pourra consulter :

- quelle différence y a-t-il entre un psychologue, une physicienne et une mathématicienne?

http://images.math.cnrs.fr/ Quelle-difference-y-a-t-il-entre.html 


\section{NOTES}

1. Le baguenodier est un instrument formé d'anneaux enchevêtrés dans une navette qu'il s'agit de séparer du système des anneaux. Ce mécanisme, véritable casse-tête, servait de serrure aux paysans : ils pensaient ainsi se prémunir des voleurs, en raison de sa complexité et du temps qu'il fallait pour le défaire, ce qui permettait de prendre le voleur sur le fait.

2. «Si les gens ne croient pas que les mathématiques sont simples, c'est seulement parce qu'ils ne réalisent pas combien la vie est compliquée !»

\section{RÉSUMÉS}

Pour s'échapper du stéréotype du scientifique enfermé dans sa tour d'ivoire, un petit nombre de mathématicien-ne-s s'emploient régulièrement à faire entrer leur discipline dans les foyers et à nourrir leur point de vue à partir du dialogue engagé avec un public très diversifié : du plus captif (les enfants) au moins concerné (une grande majorité d'adultes dont des chercheurs eux-mêmes qui ne voient pas l'utilité d'une telle démarche). Le fil conducteur de certaines de leurs actions de popularisation et de communication : le jeu. François Sauvageot nous livre le dessous des cartes...

\section{AUTEUR}

\section{FRANÇOIS SAUVAGEOT}

François Sauvageot est mathématicien au laboratoire Jean-Leray. 